ISBN 978-93-84468-86-6

2016 International Research Conference on Social Sciences, Humanities and Interdisciplinary Studies

(RCSSHIS-2016)

Pattaya (Thailand) Dec. 16-17, 2016

\title{
Work Performance Motivation of Chinese teachers of Chinese Language teaching agency in Bang $\mathrm{Na}$ district
}

\author{
Dr. WatcharaYeesuntes \\ Ms. YuanXiaomeng \\ watchara.yee@kbu.ac.th \\ Post gaduat school of business administration \\ Kasembundituniversity,Bangkok
}

\begin{abstract}
The purpose of this study was to determine work performance motivation of Chinese teachers from Chinese Languageteaching agency in Bang Na district. The samples were selected from 110 Chinese teachers of Chinese Language teaching agency in Bang Na district by using a check list and rating scale questionnaire as a tool. Statistical application used for data analysis was percentage, mean, standard deviation, T-test, One-way Anova, and regression analysis.

Findings indicated that most of respondents were females, age between 21-25 years old, single marital status, bachelor degree graduated, monthly income over 20,000 baht, teaching period 1-3 years, factors of work performance motivation were in high level consisted of career status, job achievement, relationship with colleagues, job security, job description, career advancement opportunity, private life, and company policy and management. As for factors of performance motivation were in medium level consisted of relationship with superiors, appropriate work environment, relationship with subordinate, salary and compensation, social acceptant, job responsibility, and company policy and management. Factors of personal different, such as gender, age, marital status, level of education, monthly income effect work performance motivation of Chinese teachers. Work performance motivation factors related to job satisfaction of Chinese teachers at Chinese Language teaching agency in Bang Na district revealed that company managerial policy and administration defines the duty of each person correctly, relationship with managers who were pleasurable and gave advises to employees regarding work on any occasions with statistical significant level 0.05 .

Recommendations from this study were that Chinese Language teaching agency in Bang Na district should respect and give priority to Chinese teachers, the delivery of work by the deadline, recruit Chinese teachers who can work as a team and respect other,be confident that the agency well established, assign job to teachers talent and proximity to teachers home, and administrator with managerial skill.
\end{abstract}

Keywords: Motivation, performance

\section{Introduction}

Welfares, compensation, job training, work environmentas well as their colleagues adversely affects the work performance or not or how. The work performance, however, is the work related activities expected of an employee and how well those activities were executed. Many business personnel directors assess the job performance of each employee on an annual or quarterly basis in order to help them identify suggested areas for improvement.The employee expectationis when the person making the demand in action and pushes toward a right direction to achieve the desiredor not. It cannot be denied that the environment of the work place, colleagues, job training, does not affect work in the long term. When employees feel unsatisfied with any reasons would directly impact the objection and achievements of the company, because the employees are not 
satisfied with situations. Therefore, the impact that inevitably affect services directly and inevitably harm the overall image of the organization, may be employees show other expressions, such as absentee, tardiness, unfaithfulness, and unhappy with their works.

Employees work performance efficiently are depend upon work performance motivation, such as organization reputation, self-esteem, work assigned, loyalty, and career advancement, and appropriate salary with compensation.(Siriporn Umpaisri,2547: 1-2)

As mentioned above, it shows the importance of work performance motivation in the Organization in order to support employees to work in anenjoyable,appropriateness environment, providing encouragement, wisdom, and receive the most successful result as expected by company. For the reasons that stated, researcher was interested in determine the factors affecting work performance motivation of Chinese teachers from Chinese Language teaching agency in Bang $\mathrm{Na}$ district and results will recommend to administrative officersin order to develop company managerial policy and receive better outcome in managing the agency.

\section{Literature review}

Two-factor theory and concepts of Frederick Herzberg's Two-Factor Theory

Two-factor theory distinguishes between:

1. Motivators (e.g. challenging work, recognition for one's achievement, responsibility, opportunity to do something meaningful, involvement in decision making, sense of importance to an organization) that give positive satisfaction, arising from intrinsic conditions of the job itself, such as recognition, achievement, or personal growth and

2. Hygiene factors (e.g. status, job security, salary, fringe benefits, work conditions, good pay, paid insurance, vacations) that do not give positive satisfaction or lead to higher motivation, though dissatisfaction results from their absence. The term "hygiene" is used in the sense that these are maintenance factors. These are extrinsic to the work itself, and include aspects such as company policies, supervisory practices, or wages/salary. Herzberg often referred to hygiene factors as "KITA" factors, which is an acronym for "kick in the ass", the process of providing incentives or threat of punishment to make someone do something.

According to Herzberg, hygiene factors are what causes dissatisfaction among employees in a workplace. In order to remove dissatisfaction in a work environment, these hygiene factors must be eliminated. There are several ways that this can be done but some of the most important ways to decrease dissatisfaction would be to pay reasonable wages, ensure employees job security, and to create a positive culture in the workplace. Herzberg considered the following hygiene factors from highest to lowest importance: company policy, supervision, employee's relationship with their boss, work conditions, salary, and relationships with peers. Eliminating dissatisfaction is only one half of the task of the two factor theory. The other half would be to increase satisfaction in the workplace. This can be done by improving on motivating factors. Motivation factors are needed to motivate an employee to higher performance. Herzberg also further classified our actions and how and why we do them, for example, if you perform a work related action because you have to then that is classed as "movement", but if you perform a work related action because you want to then that is classed as "motivation". Herzberg thought it was important to eliminate job dissatisfaction before going onto creating conditions for job satisfaction because it would work against each other.

\section{The Purpose of the Study}

1. To study the work performance motivation factors of Chinese teachers in Chinese Language teaching agency in Bang $\mathrm{Na}$ district

2. To study the satisfaction in the work performance of Chinese teachers in Chinese Language teaching agency in Bang $\mathrm{Na}$ district

\section{Conceptual Framework of the Study}

Researcher used the two-factor theory concepts in the Organization as a guideline in determining the conceptual framework in this study ofwork performance motivation of the Chinese language teachers atChinese Language teaching agency in Bang Na district, as follows: 


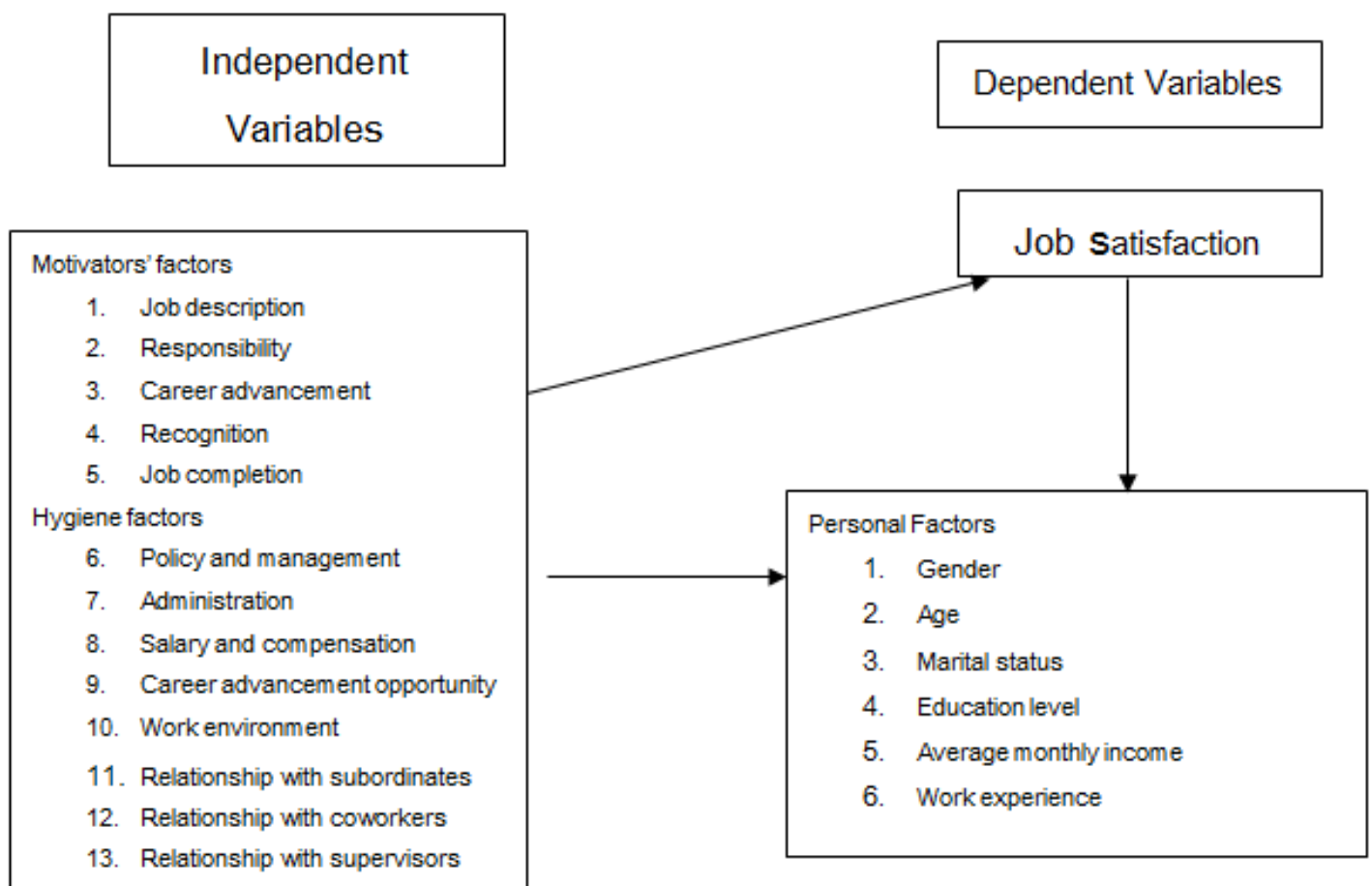

\section{Research Methodology}

\section{Sample of selection}

The samples were 150 Chinese teachers from Chinese Language teaching agency in Bang Na district

\section{Data collection procedure}

The questionnaires were distributed to sample of 109Chinese teachers from Chinese Language teaching agency in Bang $\mathrm{Na}$ district. A total of 109 usable questionnaires were returned back to the researcher, yielding a 100 percent response rate and no missing data.

TABLE I Hypothesis testing revealed that the importance of work performance motivation of Chinese-language teachers at Chinese Language teaching agency in Bang Na district classified bygender

\begin{tabular}{|l|c|c|c|c|c|c|c|}
\hline \multicolumn{1}{|c|}{ Gender } & $\mathrm{N}$ & Mean & S.D. & $\mathrm{t}$ & $\mathrm{df}$ & Sig. & test \\
\hline Male & 33 & 3.76 & 0.169 & 0.978 & 107 & 0.358 & No different \\
\hline Female & 76 & 3.59 & 0.176 & & & & \\
\hline
\end{tabular}

Hypothesis testing revealed thatthe importance of work performance motivation of Chinese-language teachers at Chinese Language teaching agency in Bang Na district classified by gender with a value equal to 0.358 which wasgreater than the statistical significance 0.05 level, shows that the test agreed withhypothesis $\mathrm{H} 0$, that meanthe factors of personal different as gender were not difference with the importance of work performance motivation of Chinese-language teachers at Chinese Language teaching agency in Bang Na district.

TABLE II Hypothesis testing revealed that the importance of work performance motivation of Chinese-language teachers at Chinese Language teaching agency in Bang Na district classified by age.

\begin{tabular}{|c|c|c|c|c|c|c|}
\hline Work motivation & SS & df & MS & F & Sig. & Test \\
\hline Between group & 2.072 & 3 & .691 & 1.051 & .373 & No different \\
\hline Within group & 68.974 & 105 & .657 & & & \\
\hline Total & 71.046 & 108 & & & & \\
\hline
\end{tabular}

Hypothesis testing revealed that the importance of work performance motivation of Chinese language teachers at Chinese Language teaching agency in Bang $\mathrm{Na}$ district classified by age with a sig. value equal to 0.373 which was greater than the statistical significance 0.05 level, shows that the test disagreed with hypothesis $\mathrm{H} 0$, that mean the factors of personal different as age were not difference with the importance of work performance motivation of Chinese-language teachers at Chinese Language teaching agency in Bang Na district. 
TABLE III Hypothesis testing revealed that the importance of work performance motivation of Chinese-language teachers at Chinese Language teaching agency in Bang $\mathrm{Na}$ district classified bymarital status.

\begin{tabular}{|c|c|c|c|c|c|c|}
\hline Work motivation & SS & df & MS & F & Sig. & Test \\
\hline Between group & 9.626 & 2 & 4.813 & 8.142 & $.001 *$ & No different \\
\hline Within group & 63.247 & 107 & .591 & & & \\
\hline Total & 72.873 & 109 & & & & \\
\hline
\end{tabular}

* The statistical significance level 0.05

Hypothesis testing revealed that the importance of work performance motivation of Chinese language teachers at Chinese Language teaching agency in Bang $\mathrm{Na}$ district classification by marital status with a value of significant equal to 0.001 which was less than the statistical significance 0.05 level showed that the test disagreed with hypothesis $\mathrm{H} 1$, that mean the factors of personal different as marital status were effected with the importance of work performance motivation of Chinese-language teachers at Chinese Language teaching agency in Bang Na district.

TABLE IV Hypothesis testing revealed that the importance of work performance motivation of Chinese-language teachers at Chinese Language teaching agency in Bang $\mathrm{Na}$ district classified by level of education.

\begin{tabular}{|c|c|c|c|c|c|c|}
\hline Work motivation & SS & df & MS & F & Sig. & Test \\
\hline Between group & .026 & 2 & .013 & .019 & .981 & No different \\
\hline Within group & 71.020 & 106 & .670 & & & \\
\hline Total & 71.046 & 108 & & & & \\
\hline
\end{tabular}

Hypothesis testing revealed that the importance of work performance motivation of Chinese language teachers at Chinese Language teaching agency in Bang Na district with a value of significant equal to 0.981 which was less than the statistical significance 0.05 level showed that the test disagreed with hypothesis $\mathrm{H} 0$, that mean the factors of personal different as level of education were not affected with the importance of work performance motivation of Chinese-language teachers at Chinese Language teaching agency in Bang Na district.

TABLE V Hypothesis testing revealed that the importance of work performance motivation of Chinese-language teachers at Chinese Language teaching agency in Bang Na district classified by average monthly income

\begin{tabular}{|c|c|c|c|c|c|c|}
\hline Work motivation & SS & df & MS & F & Sig. & Test \\
\hline Between group & 2.143 & 3 & .714 & 1.089 & .357 & No different \\
\hline Within group & 68.903 & 105 & .656 & & & \\
\hline Total & 71.046 & 108 & & & & \\
\hline
\end{tabular}

Hypothesis testing revealed that the importance of work performance motivation of Chinese language teachers at Chinese Language teaching agency in Bang Na district with a value of significant equal to 0.357 which was less than the statistical significance 0.05 level showed that the test disagreed with hypothesis $\mathrm{H} 0$, that mean the factors of personal different as average monthly income were not affected with the importance of work performance motivation of Chinese-language teachers at Chinese Language teaching agency in Bang Na district.

TABLE VI Hypothesis testing revealed that the importance of work performance motivation of Chinese-language teachers at Chinese Language teaching agency in Bang Na district classified by work experience.

\begin{tabular}{|c|c|c|c|c|c|c|}
\hline Work motivation & SS & df & MS & F & Sig. & Test \\
\hline Between group & 4.443 & 3 & 1.481 & 2.335 & .078 & No different \\
\hline Within group & 66.603 & 105 & .634 & & & \\
\hline Total & 71.046 & 108 & & & & \\
\hline
\end{tabular}

Hypothesis testing revealed that the importance of work performance motivation of Chinese language teachers at Chinese Language teaching agency in Bang Na district with a value of significant equal to 0.357 which was less than the statistical significance 0.05 level showed that the test disagreed with hypothesis $\mathrm{H} 0$, that mean the factors of personal different as work experience were not affected with the importance of work performance motivation of Chinese-language teachers at Chinese Language teaching agency in Bang Na district.

\section{Summary of Study Results}

Findings indicated that most of respondents were females, age between 21-25 years old, single marital status, bachelor degree graduated, monthly income over 20,000 baht, teaching period 1-3 years 
The work performance motivation of Chinese language teachers atChinese Language teaching agency in Bang $\mathrm{Na}$ district revealed that the average picture of opinion was high level

Details as follow:

Job description aspect: findings indicated that the average picture of opinions regarding the important of work performance motivation of Chinese Language teacher were at high level with detail, such as work assignment suitable with their skills, job responsibility acceptableper job description.

Job responsibility aspect: findings indicated that the average picture of opinions regarding the important of work performance motivation of Chinese Language teacher were at medium level with detail, such as responsibility with assigned job, able to resolve problem by themselves, independent decision making so that each employee could learn and develop at their full capacity, and organization assigned job to employees with flawless procedures.

Career advancement aspect:findings indicated that the average picture of opinions regarding the important of work performance motivation of Chinese Language teacher were at medium level with detail, such as career advancement opportunity and chance to get training for position advancement.

Recognitions aspect: findings indicated that the average picture of opinions regarding the important of work performance motivation of Chinese Language teacher were at medium level with detail, such as appreciation of employees, accepted employees opinions, decision, and admired by colleagues.

Work completion aspect: findings indicated that the average picture of opinions regarding the important of work performance motivation of Chinese Language teacher were at high level with detail, such as complete work assignment on schedule, able to solve problem when arise, and able to work according to company policy. Company policy and management aspect: findings indicated that the average picture of opinions regarding the important of work performance motivation of Chinese Language teachers were at medium level with detail, such as a measures for assignments of each personnel appropriate to the knowledge and consistent with the position, defines the duties of each personnel clearly and accurately, written regulations with policies and guidelines that are clearly.

Administration aspect: findings indicated that the average picture of opinions regarding the important of work performance motivation of Chinese Language teachers were at medium level with detail, such as supervisors were able to manage with knowledge in managerial skill andwilling to advise and supervise equally and fairly to all employees.

Salary and compensation aspect: findings indicated that the average picture of opinions regarding the important of work performance motivation of Chinese Language teachers were at medium level with detail, such as satisfy with issues as follow; income, sick leave, bonus, medical benefit, and overtime pay.

Career advancement opportunity aspect: findings indicated that the average picture of opinions regarding the important of work performance motivation of Chinese Language teachers were at medium level with detail, such as assigned job to employees'ability and skill, career advancement supportive, and career advancement training. Work environment aspect: findings indicated that the average picture of opinions regarding the important of work performance motivation of Chinese Language teachers were at medium level with detail, such as safety, appropriated temperature, proper lighting, modern working equipment, and proportion work area.

Relationship with subordinates aspect: findings indicated that the average picture of opinions regarding the important of work performance motivation of Chinese Language teachers were at medium level with detail, such as teach and counselwith approachable in all occasions.

Relationship with colleague aspect: findings indicated that the average picture of opinions regarding the important of work performance motivation of Chinese Language teachers were at high level with detail, such aswork as a team with admiration and friendly atmosphere.

Relationship with supervisors aspect: : findings indicated that the average picture of opinions regarding the important of work performance motivation of Chinese Language teachers were at medium level with detail, such as respectable relationship with managers, resolve problem together as a team, and get advice from managers at all occasions.

\section{Suggestions for Future Research}

Recommendations from this study were that Chinese Language teaching agency in Bang Na district should respect and give priority to Chinese teachers, the delivery of work by the deadline, recruit Chinese teachers who 
can work as a team and respect other, be confident that the agency well established, assign job to teachers talent and proximity to teachers home, and administrator with managerial skill.

\section{References}

[1] JirapornKhwanbua. (2546). The factors that affect the work motivation of Organization Employees: group case study. Tipco Corporation, Thesis, Master of Education, graduate school, University of the Thai Chamber of Commerce Publisher.

[2] ChukairtYimpoung. (2554). Work performance motivation: Case study, Bangkok Glass Co., Ltd., Patumthani Province, Thesis, Master of Education, Technology RatchamongkolTanyaburi University

[3] ChutimaChanphong. (2539). Work satisfaction of Water Department District employees: Case Study, Water Department district 6, 7, and 8. Thesis, Master of Public Administration, Kasetsart University.

[4] ChotikaRaso. (2555). Work performance motivation of personnel at RatchaphatNakornSawan University, Thesis, Master of Education, Srinakarinviroj University Publisher.

[5] NatthaGreehirun. (2550). Study of work satisfaction of personnel at Srinakarinviroj University, Thesis, Master of Education, Srinakarinviroj University publisher.

[6] PathomvongSrihasena. (2557). Work performance motivator of TambonKainuenvong Municipality employees, TambonBanggaja, Chantaburi Province, Thesis, Master of Education, Burapha University publisher.

[7] PradubKhanthongtip. (2536). Work satisfaction of teachers at Lopburi Municipality, Thesis, Naraesuan University publisher.

[8] PreyapornVonganutaroj. (2548). Instructional supervision, Bangkok, Enhance Media Center Publisher.

[9] PraepanTooktam. (2546). Out patient satisfaction of service at Prajomklao Hospital, Petchaburi Province, Srinakarinviroj University publisher.

[10] SiriwanSaerirat and Associate. (2550). Management and Organizational behavior, Thera film and Chitext publisher.

[11] SiripornUmpaisri. (2547). Factors effecting work performance motivation of toll collectors of Special high way system of Thailand, Thesis, Master of Public Administration, National Institute of Development Administration, publisher.

[12] SuraHeebosot. (2540). Work performance satisfaction of Administrative officers of Thailand Telephone organization, Thesis, Master of Education, Srinakarinviroj University publisher.

[13] SomyotNawgari. (2533). Management of Excellence, (3rd edition), Thammasart University, Publisher.

[14] ArtittayaSaenavong. (2555). Factors of work performance motivation of operational and Supervisors' level at Communication Technology department, M. University, Thesis, Master of Education, University of the Thai Chamber of Commerce Publisher.

[15] IssariyaRattakitvijan. (2557). Factors of work performance motivation of secondary school Teachers, district 17 (Chantaburi-Trad), Thesis, Master of Education, Burapha University, publisher.

[16] AmornratChukeaw. (2555). Work performance motivation of Ummarit and Associate Co., Ltd., Employees, Thesis, Master of Education, Thaksin University, publisher.

[17] Herzberg, Frederick; Mausner, Bernard; Snyderman, Barbara B. (1959). The Motivation to Work (2nd ed.). New York: John Wiley. ISBN 0471373893

[18] Hackman, J. Richard; Oldham, Greg R. (August 1976). "Motivation through the Design of Work: Test of a Theory". Organizational Behavior and Human Performance. 16 (2): 250-279. Doi:10.1016/0030-5073(76)90016-7. OCLC 4925746330.

https://doi.org/10.1016/0030-5073(76)90016-7

[19] Herzberg, Frederick (January-February 1968). "One More Time: How Do You Motivate Employees?". Harvard Business Review. 46 (1): 53-62. OCLC 219963337.

[20] "Herzberg's Motivation-Hygiene Theory (Two Factor Theory)". NetMBA.com. Retrieved December 9, 2014.

[21] "Herzberg's Motivators and Hygiene Factors". Mindtools.com. Retrieved December 2, 2014 